eins, das er aus eigener Anschauung kennt, beschränken würde. Er ist gerade rechtzeitig geboren, um wachen Sinnes, aber ohne Vorbelastung den Schock erlebt zu haben, den die Öffnung einer abgetakelten deutschen Wissenschaft gegenüber den USA nach 1945 bedeutet haben muss. Diese Umwälzung uns nahezubringen wäre deshalb so lehrreich, weil eine ganz ähnliche sich gegenwärtig vor unseren Augen abspielt bei der Konfrontation der «östlichen» mit der «westlichen» Wissenschaft. Wie langwierig solche Aufholjagden sein können, dafür liefert das vorliegende Buch, ungewollt, einen Beleg.

Jean Lindenmann

\title{
Zur Pathographie Friedrich Nietzsches
}

Das umfangreiche Buch von Pia Daniela Volz* - als Dissertation am Medizinhistorischen Institut der Tübinger Universität entstanden - besteht aus zwei Teilen: einer Abhandlung über Nietzsches Krankheit(en) vor und nach seinem Zusammenbruch 1889 bis zu seinem Tod 1900 und einem umfangreichen Anhang mit den wichtigsten Dokumenten zu Nietzsches Krankengeschichte und der Bibliographie der pathographischen Literatur bis in die Gegenwart.

Von zentraler Bedeutung ist sicher die Publikation der beiden vollständigen Krankengeschichten aus der Zeit der Hospitalisierung in der Basler «Irrenanstalt Friedmatt» (1.-7. Jan. 1889) und der «Irren-, Heil- und Pflegeanstalt zu Jena» (8.1.-24.3.1890). Schon in Basel hatte Ludwig Wille aufgrund des klinischen Bildes die Diagnose einer Progressiven Paralyse, d.h. einer syphilitischen, organischen Psychose gestellt, die in Jena von Otto Binswanger und Theodor Ziehen bestätigt wurde.

$\mathrm{Zu}$ den Krankengeschichten kommen verschiedene Dokumente, vor allem Briefe der Mutter und der Schwester und Berichte von Freunden, die Nietzsches letzte zehn Lebensjahre als Pflegefall, zunächst bei der Mutter in Naumburg, dann nach ihrem Tod 1897 bei der Schwester in Weimar, beleuch-

* Pia Daniela Volz, Nietzsche im Labyrinth seiner Krankheit. Eine medizinischbiographische Untersuchung. Würzburg, Könighausen \& Neumann, 1990. 4 Bl., III, 577 S. III. DM 68,- . ISBN 3-88479-402-7. 
ten. Alle diese Dokumente zusammen ergeben ein dichtes und packendes Bild von Nietzsches Situation und seinem persönlichen und gesellschaftlichen Umfeld, in welchem er selbst vom Zeitpunkt seines akuten Zusammenbruchs an eine zwar zentrale, aber notgedrungen nur passive Rolle spielte.

Nach und nach enthüllen sich aus diesen Dokumenten alle möglichen ernsthaften, tragischen und komischen Figuren von Nietzsches Lebensdrama, die die Spannweite des ganzen menschlichen Verhaltens ausloten: Da ist er selbst als passiver Mittelpunkt, das gefeierte Genie und der Verkünder des Übermenschen, der durch seine Krankheit mit der totalen Hilflosigkeit und Ohnmacht konfrontiert wird und sie nur durch peinliche Grössenideen und erotische Phantasien bewältigen kann («Ich werde Euch gutes Wetter machen»; «Zuletzt bin ich Friedrich Wilhelm IV. gewesen», «Heute nacht habe ich mit 24 Huren geschlafen», etc.). Dann ist da die Mutter, die ihrem «Herzenssohn», dem grossen Philosophen und Schriftsteller, die Kartengrüsse an die Schwester diktiert, die voll Stolz und Hoffnung erglüht, als er in völliger Verkennung seiner Lage deliriert, er halte Vorlesungen im Hörsaal der Jenaer Irrenanstalt, die bei gemeinsamen Spaziergängen, sobald Spaziergänger vorbeikommen, anfängt, laut Gedichte zu rezitieren, um die sinnlosen Laute ihres Sohnes zu übertönen, die ihm philosophische Schriften vorliest, von denen sie selbst nichts versteht, «um seinen Geist wiederzuerwecken», - kurz, die keine Mühe und keine List mütterlicher Fürsorge scheut, in der Hoffnung, ihren in kleinkindliche Passivität und Pflegedürftigkeit zurückgesunkenen Sohn als «grossen Mann» zu erhalten und zurückzugewinnen. Da ist der Verehrer Nietzsches Dr. Langbehn, der als Anwalt und Retter des in der Anstalt «Gedemütigten» und «Eingesperrten» auftritt und sich dann in seinen Briefen als kaltblütiger Profiteur und brutaler Antisemit entpuppt, der einzig auf die Vormundschaft und das Erbe seines «Idols» spekulierte. Und da sind die Ärzte, die dem Druck der Verwandtschaft und der Öffentlichkeit jeweils auf ihre eigene Weise mehr oder weniger standhalten oder zu profitieren suchen: Otto Binswanger, der Direktor der Anstalt in Jena, behandelte den berühmten Patienten jeweils gratis und wurde von der Mutter mit einer besonders schön gebundenen Ausgabe der Werke entschädigt. Wernicke verlangte für eine Konsultation, die in den letzten Lebensjahren in Weimar erwogen wurde, 800 Mark, während Kraepelin nur für $3000 \mathrm{zu}$ haben gewesen wäre. Schliesslich kam Theodor Ziehen aus Jena und stellte 60 Mark in Rechnung. (Ein Verpflegungstag in der Anstalt kostete DM 2,50).

Und da ist natürlich die bekannte Schwester, Elisabeth Förster-Nietz- 
sche, die die Bemühungen der Mutter in noch wesentlich übersteigertem Masse fortsetzt und sich den Aufbau und die Verwaltung einer NietzscheLegende zur Lebensaufgabe macht. So setzte sie sich vehement - und erfolgreich - für die Meinung ein, dass die Erkrankung des Bruders nicht auf eine syphilitische Ansteckung, sondern allein auf ein «Übermass geistiger Arbeit» und den Gebrauch des (Schlafmittels) «Chloral in stärksten Dosen» zurückzuführen sei. Sie gestaltete die Besuche bei ihrem Bruder zu einem anscheinend mystischen Erlebnis, und sie liess selbst die Totenmaske fälschen, um das Bild des Bruders dem Wunschbild anzugleichen: Die gebogene Nase musste begradigt und die schlaff herabhängende, durch einen Schlaganfall gelähmte rechte Gesichtshälfte der linken angeglichen werden.

Wer nun von der medizinhistorisch-biographischen Untersuchung von Pia Daniela Volz eine Klärung der Frage erwartet, warum sich trotz aller Dokumente und trotz der Bemühungen von nahezu hundert Jahren Forschung verschiedenster Disziplinen um Nietzsches Krankheit bis heute noch verschiedene, sich widersprechende Legenden winden, - und warum sich etwa für Nietzsches Zustand die (von Rudolf Steiner geprägte) poetische Metapher der "geistigen Umnachtung» bis heute allgemein erhalten oder durchgesetzt hat, während der betreffende Zustand damals wie heute noch gewöhnlich prosaisch mit «Demenz» bezeichnet wird -, der wird zunächst verwirrt und schliesslich geduldig und neugierig oder einfach enttäuscht und zornig werden: Pia Volz versteht ihre umfangreiche Arbeit nämlich erst als Vorarbeit für eine geplante Dokumentation «Der kranke Nietzsche (18891900)», wobei ihr die Auswertung der über 120 Titel der bisher erschienenen pathographischen Literatur "weniger ergiebig» erscheint als die Erschliessung neuer historischer Quellen, wie z. B. der Briefwechsel der Freunde und der Familie, der Zeitungs- und Hotelrechnungen Nietzsches etc. Im Verlauf der Lektüre ihrer Untersuchungen erhärtete sich mir jedoch der Verdacht, es handle sich dabei nur um einen neuen Versuch, die unermüdliche Arbeit der Mutter und der Schwester mit etwas veränderten Vorzeichen fortzuführen: Wurden Mutter und insbesondere Schwester nicht müde, das «Genie» des Sohnes und Bruders gegen das allzumenschliche Fatum einer grausamen, mit dem Odium sexueller Ausschweifung, Grössenwahn und geistiger Schwäche verbundenen Krankheit zu verteidigen, so gesteht Pia Volz ihm inzwischen doch wenigstens die sexuelle Aktivität zu. Was den Grössenwahn betrifft, schliesst sie sich jedoch ganz den krassen «Erklärungen» der Schwester an: Wenn Nietzsche z. B. beim Eintritt in die Klinik Basel im Zustand manisch-euphorischer Erregung den Ärzten verspricht: «Ich werde Euch 
gutes Wetter machen», so sei diese Äusserung nichts anderes als eine Reminiszenz aus seiner Kindheit, als er einmal Zeuge wurde, wie schlechtes Wetter die Ernte von Bauersleuten in Frage stellte, und er damals «in der Güte seines Herzens» den Bauersleuten gutes Wetter versprochen habe. In Volz' Zusammenfassung von Nietzsches Krankengeschichte ist demnach kein Grössenwahn (ganz entgegen zahlreichen Belegen in den Aufzeichnungen der Ärzte), sondern nur Verfolgungswahn genannt. Irritierend auch der (zwar aus einer Sekundärquelle zitierte Kommentar) zu einer 1898 entstandenen kleinen Genre-Plastik «Nietzsche im Krankenstuhl»: die Plastik sei dem Wunsch des Publikums nach «Vermenschlichung, d.h. Verharmlosung des Genius» entgegengekommen. Als sei die Vermenschlichung des Genius zu fürchten, und nicht etwa die Verharmlosung des Geniekults! So nimmt man auch plötzlich wahr, dass für das Titelbild nicht die Original-Totenmaske, sondern ein entsprechendes, aber «geschöntes» Bildnis mit gerader Nase gewählt wurde.

Vertieft sich der Leser oder die Leserin, auf diese Weise verunsichert, selbst in die einschlägige Lektüre über das Erscheinungsbild der Progressiven Paralyse vor der Entdeckung der spezifischen Untersuchungsmethoden und der erfolgreichen Penizillintherapie, so beginnt sich bald manches zu klären: Mir jedenfalls kam schlagartig eine Erklärung für den bombastischen, weit über die Mundpartie bis über das Kinn hinausragenden Schnurrbart Nietzsches, - wo doch der Haarschneider so regelmässig ins Haus kam und eine solche Bartpracht wohl kaum der nötigen (Mund-)Pflege des halbseitig Gelähmten dienlich sein konnte: Sollte dieses herausragende Zeichen der Männlichkeit etwa die für den Paralytiker so bezeichnende, jeweils im schroffen Gegensatz zur oberen Gesichtshälfte stehende, schlaffe und den Eindruck von Willenslosigkeit vermittelnde Mundpartie verdekken?

Hätte wohl Nietzsche selbst zu Beginn seiner Erkrankung es je für möglich gehalten, dass gerade seine Krankheit dazu dienen könnte, sein Werk und seine Person mit dem Nimbus des Numinosen und der Unsterblichkeit auszuzeichnen? Und wie ist es möglich, dass eine so plump inszenierte Maskerade mächtiger Männlichkeit über die Zeit von zwei Weltkriegen und über hundert Jahre Forschung hinaus Bestand haben kann?

Angela Graf-Nold 